\title{
How Does the Accuracy of ICD Coding Influence for Resource Consumption Under the Casemix Based Evaluation
}

\author{
Homare Sakai $^{1)}$, Keiji Muramatsu ${ }^{1)}$, Shinichiro Kobori ${ }^{1)}$, Shinya Matsuda ${ }^{1,2)}$, \\ Makoto Anan ${ }^{3)}$, Kenshi Hayashida ${ }^{2)}$, Tatsuhiko Kubo'), Yoshihisa Fujino'), \\ Kenji Fujimori ${ }^{4}$, Kiyohide Fushimi ${ }^{5)}$ \\ 1) Department of Preventive Medicine and Community Health, School of Medicine, University of Occupational \\ and Environmental Health \\ ${ }^{2)}$ Medical Informatics Division, University Hospital of University of Occupational and Environmental Health \\ 3) Medical Information Department, National Kyushu Medical Center \\ 4) Hokkaido University Hospital \\ 5) Health Policy and Informatics Section, Department of Health Policy, Tokyo Medical and Dental University
}

\begin{abstract}
In this article, the authors tried to clarify the importance of accurate coding for appropriate evaluation under the casemix scheme. For this study, we have extracted 28,995 stroke cases with sufficient information of ICD coding from the DPC database (1st July 2008 to 31 st October 2008). Based on this data set, we have classified the cases into following 3 types: atherothrombotic, thrombus and lacna type using the ICD coding information. Then we analyzed the differences in these 3 types for demographic factors (age), clinical outcome (ADL status at admission and discharge), and cost (charged cost based on F file). Results showed that clinical characteristics and resource consumptions have been different among the stroke type even though the 3 types (atherothrombotic, lacunar and embolic) are classified into the same base DPC (010060). Embolic type of stroke cases showed longer length of stay, heavier level of ADL decline and larger cost consumption with statistical significance $(p<0.01)$. The present results have indicated the importance of accuracy of ICD coding under the casemix based evaluation system.
\end{abstract}

Key words: DPC, casemix, ICD10, stroke, coding, Japan

\section{* Introduction}

Over the past $20 \mathrm{yr}$, many countries have introduced case-mix evaluation system. Case-mix system was developed by a research group at Yale University under the direction of Robert Fetter around 19801). Originally this system was developed to evaluate

\footnotetext{
Received: April 24, 2012

Accepted: July 3, 2012

Correspondence: H. Sakai, Department of Preventive Medicine and Community Health, School of Medicine, University of Occupational and Environmental Health, 1-1 Iseigaoka, Yahatanishi-ku, Kitakyushu, Fukuoka 807-8555, Japan; e-mail: sakai-homare@pref-nagano-hosp.jp
}

quality and utilization of hospital services. However, at a moment in time when there was increasing concern in the American Congress about the rapid increase of hospital cost, the hospital were not able to justify higher costs simply by asserting their patient were sicker than other hospital's without concrete evidences. The method classified each patient according to similarity of resource consumption pattern that were theoretically estimated by the combination of diagnosis and procedures. In order to treat the information by computer system, usually diagnosis is coded by ICD-10.

In Japan, MHLW has implemented the casemix based evaluation scheme for the acute care hospitals, 
Table 1 Average age of cases stratified by stroke type

\begin{tabular}{lccc}
\hline & $\mathrm{N}$ & Mean & $\mathrm{SD}$ \\
\hline Atherothrombotic & 12,407 & 72.8 & 11.7 \\
Lacuna & 13,966 & 72.2 & 12.5 \\
Embolic & 6,337 & 76.4 & 11.2 \\
\hline Total & 32,710 & 73.2 & 12.1 \\
\hline
\end{tabular}

$\mathrm{p}<0.01$ (univariate analysis of variance).

the DPC (Diagnosis Procedure Combination) project since $2002^{2}$. In the DPC algorithm, diagnosis, procedure, and co-morbidity/complication are three key variables for the classification. Additional information (e.g. birth weight in the case of neonatal intensive care) is also referred to in some groups. Diagnosis and co-morbidity/complication were coded following ICD10 (International Classification of Diseases 10th revision) coding scheme, and procedures are coded in the Japanese Procedure Code as defined in the fee schedule of the national health insurance system.

However, there were several problems in order to apply the ICD10 coding to claim. As ICD10 was originally developed for coding of cause of death, it is not always appropriate for clinical coding. For this reason, the Japanese clinicians tend not to pay enough attention to the accuracy of ICD coding.

In this article, the authors intend to propose the importance of accurate coding for appropriate evaluation under the casemix scheme.

\section{Material and Method}

Data for this study were extracted from the Japanese inpatient administrative claims database, the DPC database. The database was originally instituted as part of a national project to develop a Japanese case-mix classification system, which has been ongoing since 2002. The annual number of cases in the database is approximately 3 million. The database contains: i) main diagnoses, pre-existing comorbidities at admission and complications after admission which are coded with ICD-10 codes; ii) surgical procedures coded with Japanese original codes (Kcodes), operation time and the performed date; iii) discharge status (dead or alive); and iv) a list of drugs and blood products used and the dates of use. Study approval was obtained from the Institutional Review
Table 2 Average length of stay of cases stratified by stroke type

\begin{tabular}{lrcc}
\hline & $\mathrm{N}$ & Mean & $\mathrm{SD}$ \\
\hline Atherothrombotic & 12,407 & 24.3 & 21.6 \\
Lacuna & 13,966 & 21.8 & 21.4 \\
Embolic & 6,337 & 29.5 & 25.8 \\
\hline Total & 32,710 & 24.3 & 22.6 \\
\hline
\end{tabular}

$\mathrm{p}<0.01$ (univariate analysis of variance).

Boards and the Ethics Committee of The Tokyo Medical and Dental University. Given the anonymous nature of the data collection process, informed consent was not required.

For this study, we have extracted 28,995 stroke cases with sufficient information of ICD coding from the DPC database (1st July 2008 to 31 st October 2008). Based on this data set, we have classified the cases into following three types: atherothrombotic, thrombus and lacna type using the ICD coding information. Then we analyzed the differences in these 3 types for demographic factors (age), clinical outcome (ADL status at admission and discharge), and cost (charged cost based on F file).

\section{Results}

Table 1 shows the average age of cases stratified by stroke type. Thrombus cases have the oldest average age (76.4) followed by atherothrombotic type (72.8) and lacunar type (72.2) with statistically significance $(\mathrm{p}<0.01)$.

Table 2 shows the average length of stay of cases stratified by stroke type. Thrombus cases have the longest average length of stay (29.5) followed by atherothrombotic type (24.3) and lacunar type (21.8) with statistically significance $(\mathrm{p}<0.01)$.

Table 3 shows the average ADL level of cases at admission stratified by stroke type. Thrombus cases have the lowest average ADL at admission (33.1) followed by atherothrombotic type (50.2) and lacunar type (53.8) with statistically significance $(\mathrm{p}<0.01)$.

Table 4 shows the average ADL level of cases at discharge stratified by stroke type. Embolic cases have the lowest average ADL at admission (59.0) followed by atherothrombotic type (72.4) and lacunar type (75.8) with statistically significance $(\mathrm{p}<0.01)$. 
Table 3 Average ADL level of cases at addmission stratified by stroke type (Barthel index score for ADL measurement)

\begin{tabular}{lccc}
\hline & $\mathrm{N}$ & Mean & $\mathrm{SD}$ \\
\hline Atherothrombotic & 7,934 & 50.2 & 40.2 \\
Lacuna & 8,744 & 53.8 & 39.6 \\
Embolic & 3,897 & 33.1 & 39.6 \\
\hline Total & 20,575 & 48.5 & 40.6 \\
\hline
\end{tabular}

$\mathrm{p}<0.01$ (univariate analysis of variance).
Table 4 Average ADL level of cases at discharge stratified by stroke type (Barthel index score for ADL measurement)

\begin{tabular}{lccc}
\hline & $\mathrm{N}$ & Mean & $\mathrm{SD}$ \\
\hline Atherothrombotic & 7,397 & 72.4 & 36.7 \\
Lacuna & 8,014 & 75.8 & 35.3 \\
Embolic & 3,323 & 59.0 & 42.7 \\
\hline Total & 18,734 & 71.4 & 37.8 \\
\hline
\end{tabular}

$\mathrm{p}<0.01$ (univariate analysis of variance).

Table 5 Average cost of cases stratified by stroke type

$(1$ point $=10 \mathrm{JPY})$

\begin{tabular}{|c|c|c|c|c|c|c|c|c|c|}
\hline & \multirow{2}{*}{$\mathrm{N}$} & \multicolumn{2}{|c|}{ Total } & \multicolumn{2}{|c|}{ Injection } & \multicolumn{2}{|c|}{ Rediological imageing } & \multicolumn{2}{|c|}{ Rehabilitation } \\
\hline & & Mean & SD & Mean & SD & Mean & SD & Mean & SD \\
\hline Atherothrombotic & 12,407 & $93,886.0$ & $71,445.9$ & $17,359.5$ & $14,986.2$ & $6,465.2$ & $6,354.1$ & $9,766.8$ & $15,568.5$ \\
\hline Lacuna & 13,966 & $81,772.2$ & $69,703.4$ & $13,591.2$ & $14,743.2$ & $5,157.7$ & $4,475.8$ & $7,946.7$ & $13,586.8$ \\
\hline Embolic & 6,337 & $116,624.5$ & $87,976.0$ & $17,615.0$ & $18,275.2$ & $6,922.2$ & $5,496.4$ & $11,248.5$ & $17,547.8$ \\
\hline Total & 32,710 & $93,119.0$ & $75,312.0$ & $15,800.1$ & $15,694.7$ & $5,995.5$ & $5,501.6$ & $9,276.8$ & $15,231.3$ \\
\hline $\mathrm{p}$ value & & \multicolumn{2}{|c|}{$<0.01$} & \multicolumn{2}{|c|}{$<0.01$} & \multicolumn{2}{|c|}{$<0.01$} & \multicolumn{2}{|c|}{$<0.01$} \\
\hline
\end{tabular}

p-value: univariate analysis of variance).

Table 5 shows average cost of cases stratified by stroke type. Here we have calculated the cost as "charged cost", that is, fee-for-services equivalent cost estimated from the F-file. Embolic cases have the highest cost for total $(116,624.5$ points; 1 point $=10 \mathrm{JPY})$, injection $(17,615.0)$, radiological imaging $(6,922.2)$ and rehabilitation $(11,248.5)$ followed by atherothrombotic type $(93,866.0 ; 17,359.5 ; 6,465.2$; $9,766.8$, respectively) and lacunar type $(81,772.2$; $13,591.2 ; 5,157.7 ; 7,946.7$, respectively) with statistically significance $(\mathrm{p}<0.01)$.

\section{Discussion}

The present results have indicated the importance of accuracy of ICD coding under the casemix based evaluation system. It is very important to recognize that clinical characteristics and resource consumptions have been different among the stroke type even though the three types (atherothrombotic, lacunar and embolic) are classified into the same base DPC (010060). Fortunately, as the Japanese DPC based financing is per diem base, most of the differences in resource consumption are absorbed by the combina- tion of per diem tariff and length of stay. However, if we use this database for clinical evaluation, such as development of clinical indicators based on DPC database, we have to pay enough attention to the accuracy of ICD coding.

As Anan et al. ${ }^{1)}$ suggested, there are some problems for the accuracy of ICD coding in Japan. They have suggested two major causes for inappropriate ICD indexing; inadequate ability of clinical coders and intrinsic problem of ICD structures. Furthermore, Japanese clinicians rarely have good understanding of ICD 10. For this problem, there must be a good education system for all kinds of medical professionals.

For the problem rooted from the ambiguity of ICD definition itself, Anan et al. have developed a set of additional code ${ }^{1)}$. For example, DPC of lung cancer (040040) has additional codes in addition to ICD-10 representing for the main-bronchus (C34.\$), 10100: small cell carcinoma, 10101: non-small cell carcinoma, 10109: Unknown or unspecified. The ' 1 ' of the left 3 digits 101 request to announce the type of histopathology and ' 01 ' in the remaining two digits, 00 means small cell carcinoma, 01 non-small cell carcinoma, 09 histopathology unspecified. This new cod- 
ing system has been adopted to the governmental DPC research program since 2005. Using this dataset, it has become to analyze the clinical procedure in more precise way. For example, using additional code, Kuwabara et al. revealed the difference in use of pharmaceuticals between small cell lung cancer and nonsmall cell cancer ${ }^{2}$. Kuwabara's study suggested that the exactness of ICD indexing would influence on the validity of classification and economic evaluation of casemix grouping.

Considering the economic limitation of available resources for health sector, it is very important to have the good quality health information system. Without such a system, it is rather difficult to realize an appropriate resource allocation. Since 2010, MHLW started a social experimentation about clinical indicators. National Hospital Organization and Saiseikai group, for example, participated at this program ${ }^{3,4)}$. They calculate most of the indicators based on the DPC dataset.
Thus the accuracy of coding is precondition of the accountability of each hospital. In this meaning, the Japanese medical professionals must pay more attention for the ICD coding.

\section{* References}

1) Anan M, Kuwabara K, Hisatomi $Y$ et al.: ICD10 Code Modification Corresponding to DPC System in Japan, APJDM, Vol.3, 2009.

2) Kuwabara K, Matsuda S, Fushimi K, et al.: Differences in practice patterns and costs between small cell and non-small cell lung cancer patients in Japan, Tohoku J Exp Med, 217, 29-35 (2009).

3) National Hospital Organization. http:// www.hosp.go.jp/7,0,61.html (access 12 June, 2012)

4) Saiseikai. http://www.saiseikai.or.jp/saiseikai_wdm/ $\mathrm{html} /$ work/shihyou.html (access 2 June, 2012) 